\title{
El perfil triangular del documentalista digital: novedades y constantes del emisor de documentación en el periodismo digital
}

\author{
María Rubio Lacoba \\ Universidad Pontificia de Salamanca
}

\section{Resumen}

Se analiza el perfil profesional del documentalista que trabaja en el medio digital, con el fin de corroborar los rasgos que le son comunes al resto de documentalistas informativos, así como de aislar los nuevos roles demandados por el nuevo medio, el nuevo documento y el nuevo usuario. Se han utilizado las metodologías cualitativas de la observación directa no participante y las entrevistas en profundidad a lo largo de un mes de estudio. Los resultados de esta investigación revelan que el documentalista digital recupera roles tradicionales que se actualizan para adaptarse a las exigencias del nuevo medio, caso de la habilidad selectivaevaluadora o la habilidad recuperativa. Asimismo, el documentalista digital adquiere nuevas destrezas encaminadas a la gestión de la documentación, la especialización en el manejo de herramientas informáticas, la elaboración de productos informativo-documentales, la publicación de las ediciones digitales y la coordinación con el equipo de redacción. La investigación detecta en el perfil triangular del documentalista digital a un profesional híbrido capacitado para el manejo cualificado de herramientas y programas informáticos, así como para elaborar productos informativo-documentales en coordinación con los periodistas, pero, por encima de todo, se trata del profesional responsable de mantener la calidad documental que precisa actualmente el informador, en un entorno de saturación informativa e infopolución. Las tres caras de este perfil triangular, no obstante, han de fortalecerse ejercitando e incluso incrementando sus habilidades. Para ello, recomendamos que la base documental cuide escrupulosamente la habilidad selectiva y valorativa, puesto que Internet es una de sus principales fuentes de información $\mathrm{y}$, a la vez, es un entorno asfixiado por recursos desacreditados. Por su parte, el perfil tecnológico del documentalista digital exige conocer un amplio abanico de herramientas informáticas, lo cual implica la actualización constante de sus conocimientos, fruto del fomento de la formación continua promovida y favorecida por la empresa de comunicación mediante cursos de reciclaje, seminarios de capacitación técnica o congresos. Por último, el perfil periodístico podrá expandirse en

Scire. 11 : 2 (sep.-dic. 2005) 17-32. ISSN 1135-3761. 
la medida en que el propio periodista digital reconozca en el documentalista a un aliado, una parte del equipo con el que debe trabajar coordinadamente. De este modo, el documentalista podrá incluso elaborar productos informativo-documentales propios, firmados con su propio nombre, como símbolo de la visibilidad que ha de alcanzar su trabajo.

Palabras clave: Documentalista. Periódicos digitales. Perfil profesional.

\section{Abstract}

This paper studies the news librarian who works in a digital media, to corroborate some similar roles with another news librarians and, also, to identify the new roles requested for the new media, the new document and the new user. We selected a resource centre of a digital media, and there we applied two methodologies: no participant direct observation and interview in depth for a month. The results of this research show that being a digital news librarian involves traditional roles, like selecting and retrieving information, as well as new skills connected with management, software or the coordination with the rest of the newsroom. The main conclusion attained in this research is that the profile of the digital news librarian is triangular and hybrid: an expert in the software and the technologies but also in the edition of documents in coordination with the journalist. But, above all, he/she is a professional who takes care of the high quality of the documents that a journalist needs, in a context of excess of information. These three sides, however, need to be developed. For this purpose, it is necessary to look after the selection and the valuation, because Internet is one of the main sources of information and, in the same time, it is an environment with too many invalid resources. Second, the technological side requires a permanent training in the best available software for this work. Finally, the journalist side could be fulfilled when the journalist recognizes in the news librarian an ally, a necessary part of a team that needs to work in coordination.

Keywords: Information professional. Mass media. Professional profile.

\section{Introducción (1)}

Entre las muchas alteraciones que ha supuesto Internet, nos interesa especialmente aquella que ha repercutido sobre los medios de comunicación en general, y sobre la documentación que se emplea en estos, en particular. Si la reflexión en torno al periodismo digital ha resultado abundante y diversa - a veces incluso discrepante-, no podemos decir lo mismo sobre el análisis suscitado por la documentación empleada en dicho nuevo periodismo. Por ello, debido al surgimiento de un nuevo medio — el periodismo digital—, de un nuevo usuario —el periodista digital— e incluso de un nuevo mensaje — la información periodística digital—, vemos consecuentemente que los servicios de documentación han de vivir una 
adaptación necesaria, con el objeto de seguir satisfaciendo las nuevas necesidades de sus usuarios y propiciando las herramientas oportunas que permitan construir una información completa y de calidad.

De igual modo que el servicio documental digital adapta su estructura a la nueva organización del periodismo digital, el documentalista digital reinterpreta sus funciones ante la aparición de un nuevo usuario, el periodista digital, quien desarrolla ciertas habilidades documentales que le exigen un cambio de perfil, si bien no un cambio de objetivos, pues estos siguen siendo la satisfacción rápida y adecuada de las necesidades informativo-documentales de los periodistas, con el fin de elaborar una información periodística que promueva el conocimiento de los ciudadanos.

El objeto, por tanto, de este trabajo es definir el perfil del emisor de la documentación digital, un profesional cuya fisonomía se dibuja combinando el redescubrimiento de ciertas habilidades documentales tradicionales, por un lado, y el surgimiento de nuevas cualidades informativas y tecnológicas, por otro, adaptadas a la naturaleza del nuevo servicio de documentación y a las demandas del periodista digital (2).

El documentalista digital presenta un perfil triangular, un triángulo cuyos lados contornean los tres rasgos más representativos del emisor de la documentación digital. De los tres laterales del triángulo, la base siempre se sostiene sobre la faceta documental del emisor, y de esta se extienden dos lados parejos y complementarios, el periodístico y el tecnológico. Los lados y la base delimitan las diferentes habilidades profesionales que caracterizan a los documentalistas digitales; son tres prismas que, de modo armónico, reúnen integradamente las diversas actividades ligadas con las tareas documentales tradicionales, con los cometidos periodísticos y con las nuevas habilidades tecnológicas.

La estructura triangular resulta oportuna pues permite representar la interrelación constante que vincula a cada uno de los perfiles, sin que exista el solapamiento de uno sobre otro, sino la cooperación de las tres facetas profesionales. Por otro lado, el matiz de optar por que la base sea documental no es accesorio, puesto que ilustra el valor constitucional del perfil documental, el cual sostiene a los otros dos rasgos complementarios, el periodístico y el tecnológico. Por último, el diseño triangular permite contemplar un cierto tipo de actividades que, por su proximidad, se encuentran influenciadas por dos perfiles. De ahí que, como describiremos más adelante, haya tareas documentales con tintes periodísticos, o procesos periodísticos con trasfondo tecnológico, por citar algunos casos. Así pues, el triángulo que describe el perfil del documentalista digital es el siguiente:

Siguiendo la estructura triangular propuesta, nos adentramos en el estudio de cada uno de los laterales que contornean el perfil del documentalista digital, en Scire. 11 : 2 (sep.-dic. 2005) 17-32. ISSN 1135-3761. 


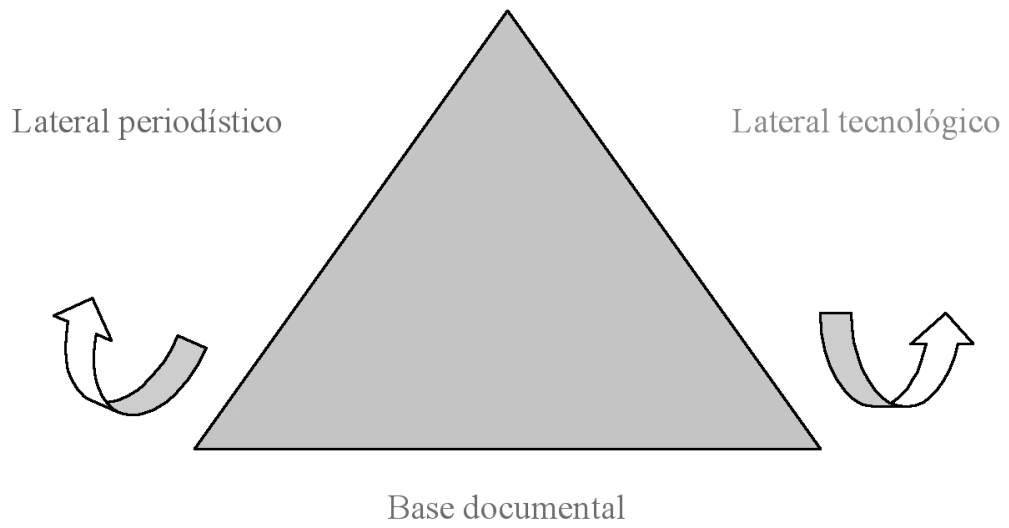

Figura 1. Perfil del documentalista digital

algunos casos semejante al de las propuestas normativas (SLA, 1996; Paul, 1997a; Galdón, 2002), y en otras circunstancias más cercano a la realidad limitada, compleja y cambiante del nuevo profesional objeto de análisis.

\section{Base documental}

Constituye el fundamento de su perfil profesional, más allá de la adaptación a las peculiaridades del servicio de documentación y a las necesidades de los periodistas. El perfil del documentalista digital se levanta sobre su base documental, como ellos mismos corroboran. Así, rodeados de tecnología y de herramientas digitales, no dudan en reconocer cuáles son las bases de su trabajo:

En cuanto al oficio del documentalista, básicamente es el mismo porque se cataloga, con diferentes herramientas, con diferentes thesaurus, pero, en esencia, catalogas para poder recuperar esa información [...]. Yo creo que el centro de documentación tiene unas labores esenciales que le son ineludibles.

Reforzando esta idea, otro documentalista comenta que

el trabajo es bastante parecido, y lo que cambia es el modus operandi, cambia tu manera de trabajar, tu tarea diaria, pero la profesión es la misma. Existe un mayor conocimiento de las nuevas tecnologías, y también cambia el enfoque: la rapidez con la que se trabaja; aquí todo lo que se pide es para ya, la inmediatez te la impone el medio. El resto sigue igual.

El perfil documental justifica y fundamenta el resto de facetas del documentalista digital, aportando soluciones tradicionales a las nuevas demandas y necesidades informativas; esto es, ante una coyuntura de exceso devaluado de la información, y donde las rutinas periodísticas se aceleran — puesto que los diarios digitales actualizan sus contenidos conforme se conocen las noticias-, los do- 
cumentalistas reinterpretan dos habilidades documentales tradicionales: la de seleccionador-evaluador de información y la de recuperador de información. Junto a esto, apreciamos cómo los papeles de gestor y miembro del equipo redaccional se incorporan como nuevas capacidades vinculadas con la faceta documental del emisor. Veamos, a continuación, en qué consiste cada una de estas tareas.

\subsection{Habilidad selectiva-evaluadora}

Únicamente a través de Internet, las redacciones digitales se encuentran inundadas de información. Si a esa catarata informativa se le añaden los datos provenientes de los medios propios, de la prensa y las revistas ajenas, de las agencias de noticias, de las emisoras de radio y las televisiones, entonces el resultado es un conjunto desmesurado de fuentes que el periodista es incapaz de criticar ni valorar.

Pero no solo la cantidad de información es perniciosa. Este crecimiento incontenible de datos suele caracterizarse, en numerosas ocasiones, por ser información no contrastada, incompleta, no autorizada o desfasada.

$\mathrm{Si}$ a la cantidad de información y a la baja calidad de esta se le añade el condicionante temporal con el que trabajan los periodistas digitales - limitados en todo momento por la actualización constante de las noticias-, entonces el papel del documentalista como rápido y eficaz seleccionador-evaluador de fuentes resulta crucial. Una actividad ligada desde el principio a las tareas documentales recobra ahora su valor ante una situación de saturación informativa y de acelerada rapidez de respuesta. Y ciertamente, los documentalistas han readaptado su habilidad evaluadora al nuevo contexto, como así constatan: "Ha habido que aprender a evaluar los contenidos que hay en la Red, discernir aquello que es realmente fiable y aporta una información interesante de aquello que son datos dudosos, que no te ofrece una fiabilidad".

Sobre los criterios de selección, así describen cuál es el procedimiento para escoger las mejores fuentes en un contexto de sobrecarga informativa:

Tenemos diversas fuentes y depende de lo que te pidan: si no son cosas concretas del periódico, que las localizas en la base de datos, tiendes a acudir a los organismos oficiales, y vas orientado por el descriptor: si es algo de economía, te vas a la web del Ministerio de Hacienda y, si no, organismos relacionados, y si no en las universidades, que suelen tener muchos enlaces; si te piden una cosa muy específica, puedes localizarlo en los enlaces de las facultades. También tienes los buscadores, como Yahoo, que para búsquedas rápidas sirven para localizar lo que deseas.

Vemos, por tanto, que se opta en primer lugar por seleccionar fuentes propias, que aseguran la fiabilidad de sus datos, fuentes oficiales, por cuanto se consideran autorizadas y legitimadas, pero también apreciamos el empleo compatible de fuentes generalistas, como los buscadores habituales, que pueden recuperar fuentes de todo tipo, si bien en ocasiones bastante alejadas de los requerimientos de la pertinencia informativa.

Scire. 11 : 2 (sep.-dic. 2005) 17-32. ISSN 1135-3761. 
No obstante, y a pesar de los criterios anteriormente señalados, los propios documentalistas también reconocen dos agujeros negros dentro de sus procesos de selección: el abuso de la intuición y la escasez de fuentes de autoridad tradicionales. Sobre el primer aspecto comentan:

\begin{abstract}
A la hora de seleccionar fuentes de Internet me guío por mi experiencia, porque llevo navegando desde el 95, desde que me puse Internet en casa, me conozco bien la Red, tengo una buena lista de favoritos actualizada que te saca de mil apuros, y ya te haces callo, y al final te guías mucho por la intuición, pero corres el riesgo de que te pases una información que no sea veraz y eso es un error que puedes ir arrastrando.
\end{abstract}

La segunda carencia es valorada de este modo:

Yo me encuentro con unas herramientas pobrísimas y me las veo y me las deseo para confeccionar un material de calidad. Lo que no podemos hacer es un material de calidad con herramientas que no lo son. Si nosotros trabajamos con Internet, yo quiero una base de datos digna y material digno para hacer cosas.

Finalmente, la tarea seleccionadora se culmina con la valoración del material seleccionado por parte del documentalista y del periodista, puesto que es este quien, en último término, determina la idoneidad del recurso documental, usándolo o desechándolo. Así, el documentalista solicita al periodista que acuda al servicio de documentación para que evalúe las fuentes recogidas y escoja aquellas que más le satisfacen. Esta selección conjunta, además, ofrece un valor añadido, en la medida en que fomenta la coordinación entre periodistas y documentalistas, el conocimiento mutuo y la integración óptima de sus actividades.

\title{
2.2. Habilidad recuperativa
}

La capacidad para recuperar información en un medio digital se encuentra condicionada por dos circunstancias propias del medio: la actualización permanente de los contenidos, que exige que la recuperación de información sea inmediata; y la habilidad documental adquirida por el periodista digital, quien es capaz de recuperar por sí mismo cierto tipo de informaciones.

Esto implica que el documentalista reinterpreta su rol de recuperador de documentación del modo que estos apuntan:

Si estás esperando a que te pidan una información, estás perdiendo el tiempo. Antes se podía hacer porque, mientras te hacían una petición, aún faltaba cierto tiempo para que saliera la edición, pero eso no pasa con Internet, en donde hay que publicar inmediatamente porque tu competencia lo está haciendo; la tienes que tener preparada.

Por lo tanto, la solicitud de información implica una rápida recuperación del documentalista, esfuerzo que demuestra la coordinación necesaria que se entabla entre periodistas y documentalistas, quienes tratan así de acompasar sus ritmos de trabajo: 
Te ha hecho trabajar a un ritmo muchísimo más rápido, primero por el tipo de usuario que tenemos, que, cuando demandan información, están demandando para ya, no para dentro de cuatro días, sino que necesitan información. Lo que también te exige este medio es que los contenidos tienen que estar en la Red lo antes posible y bien documentados, para poder recuperarlos, porque el medio lo demanda.

Por su parte, el hecho de que Internet sea una fuente de información de gran utilidad para el trabajo de los informadores, que les permite acceder rápidamente a recursos documentales, organismos o personalidades, de modo directo y satisfactorio, conduce a que el documentalista renueve su perfil tradicional de guardabarreras de la información, de intermediario entre la información y el periodista (cfr. Paul, 1997b; Salvador et al., 1998; Hernández y Caridad, 2000). Ahora, la recuperación se dirige por otros caminos:

En el mundo de los medios, los periodistas han estado buscándose su información porque es su modo de trabajo. Desde este punto de vista, son más independientes. Ahora el documentalista no ha sido un intermediario entre el periodista y la información sino más bien ha de tener controlada toda la información que ha tratado el medio para cuando haga falta.

Proporcionalmente, el incremento de la autonomía documental del periodista también lleva aparejado el descenso del número de consultas verificativas, lo cual conduce a la pérdida de la función recuperativa clásica, como reconocen los documentalistas:

El periodista dispone, por un lado, de la base de datos que le permite localizar información muy rápidamente, y por tanto, cuenta con la misma herramienta que nosotros. Además, se les ha enseñado a manejar la base de datos. Entonces, sí que es cierto que esa función ha ido perdiendo importancia, pero creo que tampoco es negativo, porque sí es cierto que el periodista, cuando necesita una información y no la localiza, al final acaba recurriendo a documentación.

En cualquier caso, y a pesar de que los periodistas sean capaces de elaborar sus propias búsquedas y recurran con menos frecuencia al servicio de documentación, los documentalistas no dudan en determinar que esta sigue siendo la tarea que más recompensas les concede: "El servicio a la gente es lo que ofrece más gratificación, la satisfacción de que la gente te pide cosas y que tú las encuentras".

\subsection{Habilidad gestora}

Fruto de la reinterpretación de la habilidad recuperativa del documentalista nace una habilidad adaptada a las nuevas necesidades de los periodistas digitales y al contexto procurado por el periodismo digital, y, en general, por la sociedad del conocimiento. La clave para controlar el caudal ingente de información que de Internet emana pasa por incentivar el perfil gestor del documentalista, que le lleva a convertirse en un profesional capacitado para evaluar la información en función

Scire. 11 : 2 (sep.-dic. 2005) 17-32. ISSN 1135-3761. 
del conocimiento de sus usuarios, organizar dicha información, controlarla y poder obtenerla por sí mismo u orientar al periodista sobre cómo y dónde localizarla.

La capacidad gestora exige un alto grado de confianza empresarial en el servicio de documentación para que disponga de recursos humanos y técnicos que favorezcan el desarrollo de esta habilidad. Junto a ello, los propios informadores han de involucrarse en la gestión de la información, colaborando estrechamente con los trabajos de los documentalistas, compartiendo la información o los recursos que conocen, informándoles de sus necesidades y prioridades informativas, y evaluando junto a ellos la calidad del servicio documental.

Sin embargo, la habilidad gestora se encuentra todavía en un proceso embrionario, puesto que ni las empresas se implican dotando de suficientes recursos ni la mayoría de los periodistas fomentan este tipo de relaciones cooperativas. Por tanto, más que una habilidad consumada, la capacidad gestora es un aspecto deseable pero aún no alcanzado, si bien no conviene olvidar que tanto el servicio de documentación digital como el documentalista digital se encuentran en la misma fase de constitución, con cambios y planes de futuro constantes, entre ellos la incorporación de tareas vinculadas con la gestión de la información:

Si ahora tuviéramos toda la gente que teníamos al principio, podríamos hacer: aparte de estar más encima de los medios para preguntarles qué necesitan, prepararles por anticipado información antes de que acontezca y, si el periodista, a la hora de hacer el tema, sabe que existe este material, lo puede utilizar. Por otro lado, se podría hacer todos los días una selección de las noticias especializadas: música, información económica, información universitaria, información general, publicidad, informática... y enviarlas mediante un boletín de noticias [...]. Desde el punto de vista de la gestión del conocimiento, se podría compartir mucho más la información, que los que estén especializados en un tema lo compartan con los demás para enriquecernos todos. Desde ese punto de vista, podríamos tener controlados todos los libros y revistas de todos los departamentos, sin que estén descentralizados, para que así estuvieran accesibles para todos.

Una de las posibles concreciones de la habilidad gestora se encuentra en el diseño y el mantenimiento de la Intranet. La Intranet se constituye como una plataforma desde la cual es posible solicitar, compartir y ofrecer todo tipo de información, promoviendo un movimiento enriquecedor de la información, así como la organización y el aprovechamiento de la información parcial que, organizada en este tipo de espacios, puede revalorizarse procurando un enriquecimiento informativo general.

\subsection{Habilidad integradora-coordinadora}

Esta última capacidad del emisor de la documentación digital discurre muy próxima al lado periodístico, puesto que existe un número de tareas compartidas por ambos profesionales que exigen una necesaria comunicación. Efectivamente, hemos detectado tareas periodísticas elaboradas por documentalistas y tareas documen-

Scire. 11 : 2 (sep.-dic. 2005) 17-32. ISSN 1135-3761. 
tales llevadas a cabo por periodistas, de ahí que la coordinación sea un elemento esencial que se encuentra a medio camino entre la base documental y el perfil periodístico. Como aseguran los documentalistas con claridad, "si el periodista hace de documentalista, el documentalista hace de periodista".

En realidad, todas las tareas anteriores cobran su sentido pleno siempre que pasen por la capacidad de coordinación recíproca entre el documentalista y el periodista. Cuanto mayor sea el flujo de comunicación entre estos profesionales, que intercambian tareas en algunos casos, mejor será la calidad de sus trabajos, puesto que los perfiles no se superpondrán y las actividades serán cooperativas y no conflictivas.

Por señalar un caso de coordinación de actividades entre periodistas y documentalistas, hemos de destacar la asignación conjunta de descriptores. En efecto, el análisis documental se está convirtiendo en una tarea repartida entre estos dos profesionales: por un lado, los documentalistas se encargan de clasificar los contenidos de las ediciones en papel, como paso previo a su publicación en la web. De esta manera, todas las noticias de la edición papel que se suben a la Red se encuentran inmediatamente analizadas por los documentalistas. Por otro lado, los periodistas digitales se encargan de ir clasificando documentalmente las noticias con las que van actualizando los diarios digitales, por lo que han tenido que familiarizarse con el manejo del thesaurus, los descriptores onomásticos, geográficos y de organismos.

Si percibimos una positiva integración en las tareas del análisis documental, también apreciamos que no se encuentra tan avanzada la coordinación en la recuperación de información. En este aspecto, es posible constatar que la capacidad de autodocumentación desarrollada por el periodista, si bien libera al documentalista para poder preparar material por anticipado y elaborar productos documentales propios, independiza en ocasiones demasiado el trabajo del periodista. Es decir, el periodista se ve capacitado para recuperar por sí mismo información, de manera que no acude con tanta asiduidad al documentalista, y desecha la documentación que este le suministra, aun cuando sean productos documentales de producción propia, que son, sin duda, un valor añadido respecto a los productos tomados de agencias u otras fuentes ajenas.

Por lo tanto, la coordinación es una habilidad recíproca que debe ser aceptada y fomentada tanto por el documentalista como por el periodista; de ahí que este puente haya de ser cruzado simultáneamente por ambos profesionales: que el periodista conozca la actividad del documentalista, la valore y la integre en su trabajo periodístico, y que el documentalista sepa de las necesidades del informador y le ofrezca material documental propio que cualifique y distinga sus informaciones.

Scire. 11 : 2 (sep.-dic. 2005) 17-32. ISSN 1135-3761. 


\section{Lateral periodístico}

Si bien la base documental sustenta, como acabamos de ver, el perfil del documentalista digital, hemos de volver al triángulo para apreciar que, a partir de ese fundamento documental, se proyectan dos nuevos lados, que contornean perfectamente al emisor de la documentación digital: el lado periodístico y el tecnológico. Atendiendo al perfil triangular, observaremos que el lado periodístico encuentra su origen en la base documental, de manera que en ningún caso busca restar funciones a las tareas propias del periodista digital. Se trata, por el contrario, de un perfil nacido del puente que se tiende entre estos dos profesionales, del conocimiento de sus actividades y de la respuesta dada desde la documentación para aliviar y mejorar el trabajo del informador.

Ciertas actividades del emisor de documentación digital están impregnadas por matices periodísticos; no en vano su contexto laboral es un medio de comunicación, su materia prima es la información periodística y su usuario principal y estrecho colaborador es el periodista digital. Estos condicionantes, en realidad, son propios de cualquier documentalista informativo, puesto que todo emisor documental adapta su trabajo a las peculiaridades del medio - ya sea prensa, radio, televisión-, a las necesidades de los usuarios y a las propiedades particulares de los documentos — desde la documentación textual hasta la sonora o la audiovisual.

Sin embargo, como resultado de la integración promovida entre los periodistas y los documentalistas, por un lado, y la liberación de ciertas peticiones verificativas ahora asumidas por los periodistas, por otro, el documentalista digital se ve capacitado para acometer actividades propiamente informativas, difundiendo productos informativo-documentales que acompañan, completan y profundizan las informaciones periodísticas. Veamos, a continuación, algunas de estas tareas.

\subsection{Elaborador de mensajes informativo-documentales}

Todos los documentalistas aprecian un cambio en las habilidades de los periodistas, que afecta a su trabajo y al cual ellos se han adaptado asumiendo nuevas actividades. Los documentalistas destacan que ahora pueden

hacer productos informativos que luego les van a venir bien a ellos: fichas, previsiones, calendarios, cronologías de desastres, biografías... Cuando ocurre un acontecimiento de última hora, hay que subir la noticia lo más rápidamente posible y también empiezas a mirar quién está poniendo la información más completa: el mapa de localización, la ficha del país, tener esos productos preparados.

Esta labor de preparación anticipada da como resultado la elaboración de una serie de productos informativo-documentales que complementan adecuadamente las noticias, incluso aquellas de las que se posee un flash informativo y que, gracias a estos apoyos, pueden subirse a la web arropadas por un entorno documental adecuado. El tiempo que antes dedicaban a localizar todo tipo de peticiones formuladas por los periodistas, ahora lo invierten 
en otro tipo de búsquedas de información más compleja, que lleva mucho más tiempo, y el periodista no dispone de ese tiempo. Por ejemplo, buscar material fotográfico para la elaboración de especiales, búsqueda de material para la elaboración de temas en profundidad... En esos casos el periodista no dispone del tiempo necesario para hacerlo. Entonces nos centramos en ese tipo de tareas.

Los productos propios de los documentalistas son la elaboración de temas y especiales, la guía de recursos web, la elaboración de fichas biográficas, geográficas y de organismos, que se emplean como complementos documentales de las noticias de última hora, o la agenda con las previsiones informativas, "con la que el periodista puede estar informado de qué previsiones y acontecimientos va a ver a corto, a medio o a largo plazo".

En cualquier caso, la asunción de nuevas tareas vinculadas con la producción documental propia se encuentra encallada en dos escollos, uno de orden histórico y otro coyuntural, esto es, el servicio documental sigue sin contar con el apoyo convencido de la empresa de comunicación y adolece de ciertas herramientas técnicas que alivien al documentalista de algunas tareas sencillas pero que nada tienen que ver con la documentación.

\subsection{Publicador de la primera edición digital}

Esta es, sin lugar a dudas, una de las actividades informativas más novedosas asumidas por el documentalista digital. De hecho, por su peculiaridad, se trata de una tarea difícil de demarcar, pues posee rasgos documentales, ya que, al mismo tiempo que se introducen los contenidos en el sistema editorial para publicarlos, se tratan documentalmente; pero también presenta un claro componente tecnológico, porque exige el manejo simultáneo de diversos programas informáticos cuyo resultado se refleja en la puesta en la Red de los diarios y los suplementos.

Sin embargo, se ha optado por encuadrarla dentro de las funciones periodísticas porque la publicación es una de las tareas esenciales de cualquier periodista, cometido que ahora comparte con el documentalista digital, que así lo justifica:

Lo que también ha cambiado en las funciones del documentalista es que se ha integrado en lo que es llevar las ediciones impresas a la Red. Antes era un trabajo que hacían los periodistas en los comienzos de las ediciones digitales y ahora es un trabajo que está asumiendo el documentalista. Y lo está asumiendo porque así se garantiza que los contenidos que se están incorporando al sistema editorial llegan perfectos, llegan en unas condiciones óptimas para que luego no haya que estar corrigiendo y rectificando.

Los documentalistas, efectivamente, son los encargados de publicar la primera edición del diario digital, al igual que editan y suben a la Red los respectivos suplementos del diario. Por su parte, los periodistas asumen la tarea de editar, de publicar y de asignar los descriptores — que, recordemos, es una tarea documentalde cuantas noticias van actualizando dicha primera edición. Vemos en la publicación y en la actualización de los diarios una nueva actividad conjuntamente

Scire. 11 : 2 (sep.-dic. 2005) 17-32. ISSN 1135-3761. 
asumida por periodistas y documentalistas, que reclama nuevamente la cooperación y la optimización de las funciones de ambos profesionales.

El proceso de edición y publicación resulta relativamente sencillo y mecánico, si bien requiere un cuidadoso y pulcro trabajo para que, a la hora de visualizar el diario digital, todo esté en su sitio y correctamente organizado. Se trata, en cierta medida, de la interpretación actualizada de la función crítico-verificadora (Galdón, 2002, pp. 72-76), dado que es el documentalista quien prefiere asegurarse por sí mismo de que la información aparezca adecuadamente en la web, sin tener que estar posteriormente enmendando fallos cometidos por otros.

No obstante, la misión de la publicación en la Red es interpretada de diferentes maneras, según lo haga el jefe de documentación o el resto de documentalistas. Para el primero, no cabe duda de que esta es una labor informativo-documental que permite controlar la calidad de la publicación de los diferentes diarios digitales, y así justifica que esta tarea "no es del todo descabellada porque está asegurando que los contenidos llegan al sistema editorial en unas condiciones óptimas, y siguiendo unas normas". Mientras, algunos documentalistas encuentran que la publicación en la Red les ha llegado por la confluencia de ciertas cuestiones coyunturales, y creen que "eso lo están haciendo documentalistas porque hacía falta gente y se tiró de documentación". Otros aprecian el fundamento documental de esta actividad, pero aseguran que debería ser un proceso mucho más mecanizado; y hay quienes consideran que esta función es, sencillamente un retroceso en su trayectoria profesional.

\section{Lateral tecnológico}

El tercer lateral que cierra el triángulo del emisor de la documentación digital es el tecnológico (3). Dicho factor, adoptado de modo general tanto por los documentalistas como por los periodistas de los medios tradicionales, se vuelve un requisito imprescindible en el caso de los profesionales cuyo medio de comunicación es un diario digital, su materia prima es la documentación digital y sus usuarios principales son periodistas digitales: "En la adaptación de las funciones del documentalista, ha habido que aprender y conocer los lenguajes que se utilizan en Internet, tipo HTML, o XML”. Ciertamente, dentro del triángulo que delimita el perfil de los documentalistas digitales aparece la imagen de un profesional con altos conocimientos tecnológicos: participa en el diseño del diario en XML, contribuye en la creación y el mantenimiento de las diferentes bases de datos, conoce y maneja los programas de tratamiento de imágenes, navega constantemente por Internet, publica en la Red los diarios digitales... Son algunas actividades cotidianas que reflejan el condicionante tecnológico de su perfil.

El factor tecnológico, no en vano, es uno de los aspectos que diferencia al documentalista digital de cualquier otro emisor de documentación, y así el documentalista digital se caracteriza por

Scire. 11 : 2 (sep.-dic. 2005) 17-32. ISSN 1135-3761. 
tener que conocer lo último que se está llevando a la Red, lo último en cuanto a formatos, en cuanto a edición de todo tipo de ficheros, lo último en cuanto a herramientas de gestión... Yo creo que eso es muy positivo y que al mismo tiempo es necesario. Y no solo nosotros, sino que los periodistas llevan el mismo bagaje; ya no se trata del periodista tradicional de "no, yo, que me enseñen mi editor, y que me digan dónde debo escribir". Ellos también investigan y se forman en cosas como convertir un tif en un $p d f$, y creo que es algo esencial, porque también estás publicando, estás llevando a la Red, luego tienes que tener esos conocimientos.

Esta nueva habilidad característica del documentalista digital no supone, sin embargo, un ocultamiento de sus funciones documentales; al contrario: como esquematiza el diseño triangular, tanto el lado tecnológico como el periodístico convergen en el enriquecimiento de la base documental. Y así lo creen:

Mi formación tecnológica no ha tapado mi progresión como documentalista y la complementa bastante bien. No podemos olvidar que esto es un medio digital, es como un documentalista que trabaja en una farmacéutica, que tiene que complementar sus conocimientos. Pues aquí pasa lo mismo, es un medio digital y además es un medio de comunicación, y nosotros hacemos también un poco de periodistas. Al final acabamos siendo un híbrido entre documentalistas-periodistas-informáticos y, si no informático, que maneje muchas herramientas informáticas.

\subsection{Experto en el manejo de herramientas informáticas}

Los documentalistas digitales se han convertido en expertos profesionales de la informática, y manejan todos los lenguajes y las herramientas que les sean necesarios para elaborar su trabajo del mejor modo.

Lo cierto es que, a pesar de que el soporte tecnológico que rodea sus trabajos resulta llamativo, ellos mismos son conscientes de que las habilidades informáticas son un sustento muy necesario pero complementario en su trabajo; quiere esto decir que son exigibles los conocimientos informáticos pero que, en último término, no dejan de ser más que herramientas que se ponen a su disposición. Lo aclaran de esta manera:

La mecanización, la herramienta no es el fin, es un medio, la herramienta tiene que servir para que tú puedas hacer algo [...]. Yo creo que la tecnología puede facilitar mucho el trabajo. Antes, si querías guardar una entrevista, tenías que hacer diez fotocopias y guardarla en diez sitios: personajes, temas... Ahora eso no; ahora ni siquiera tienes que guardar ese reportaje sino el fichero de ese reportaje, o el $p d f$, es decir el equivalente electrónico de la fotocopia.

Sin embargo, las herramientas, su elaboración y manejo, como prácticamente todo cuanto gira en torno a los servicios de documentación digital, se encuentra en un proceso de permanente cambio y evolución. De ahí que el documentalista requiera un periodo de adaptación que le exige un esfuerzo adicional:

Scire. 11 : 2 (sep.-dic. 2005) 17-32. ISSN 1135-3761. 
El documentalista tiene que conocer las herramientas, y ese es un error que tenemos en todos los trabajos, que no conocemos las herramientas lo suficiente como para pedirle al que hace esa herramienta que le haga mejoras; si lo dejas en manos de los expertos, lo que vas a tener es una aplicación comercial, que te puede servir, o que se queda corta, o que no sirve para nada [...]. Lo importante aquí es que el documentalista sepa qué hacer con las posibles herramientas que tiene a su disposición.

En último término, y del mismo modo en que los documentalistas han de coordinarse con los periodistas para establecer sinergias de trabajo e integrar sus respectivas actividades e incluso intercambiar algunas de estas, es preciso tender un nuevo puente comunicativo que coordine las labores documentales con las informáticas, pues entre ellas existen muchos puntos confluyentes. Así, el documentalista debe preguntarse cómo afecta la herramienta informática a su materia prima y al modo en que esta se presenta a sus usuarios, para trasladar dicha reflexión a los informáticos y que desarrollen herramientas verdaderamente funcionales. El resultado de esta coordinación desembocaría en un trabajo más eficiente, como desearían los documentalistas:

Yo tengo que preparar la información de tal modo que cualquier usuario, desde su puesto de trabajo, y con una herramienta adecuada, pueda servírsela él solo. Antes tenías que perder diez minutos o tres horas en buscar mano a mano o en base de datos el artículo de la revista que te piden. Ahora, el usuario tiene que poder entrar en un sitio y localizarlo, y en un momento la encuentra. Si seguimos funcionando en que seguimos metiditos en nuestro despacho, y nos vienen a buscar, estamos perdiendo las utilidades de la informática y sobre todo de las redes de comunicación.

\section{Conclusiones}

El perfil tecnológico del documentalista digital, junto con el lado periodístico, cooperan en elaborar con mayor calidad y profesionalidad su cometido documental, que, en definitiva, es favorecer que los usuarios puedan encontrar una información documental de calidad, organizada, completa y de fácil consulta. En este sentido, vemos cómo la estructura y las funciones del servicio de documentación digital y el diseño del perfil triangular del documentalista digital confluyen en la construcción de un mensaje documental con una estructura original y diferente, que aprovecha las peculiaridades del medio digital y los conocimientos de los emisores de la documentación.

Las tres caras de este perfil triangular, no obstante, han de fortalecerse ejercitando e incluso incrementando sus habilidades. Para ello, la base documental debe cuidar escrupulosamente la habilidad selectiva y valorativa, puesto que Internet es una de sus principales fuentes de información y, a la vez, es un entorno asfixiado por recursos desacreditados. Por tanto, el documentalista digital debe compaginar 
la selección de fuentes digitales y fuentes tradicionales, para cotejar información de la Red de dudosa calidad con otro tipo de fuentes que, por su trayectoria, estén suficientemente avaladas.

Por su parte, el perfil tecnológico del documentalista digital exige conocer - en coherencia con el medio y con los documentos que sostienen su trabajo- un amplio abanico de herramientas informáticas, lo cual implica la actualización constante de sus conocimientos. La formación continua del documentalista ha de ser promovida y favorecida por la empresa de comunicación, quien deberá estimular la periódica formación de sus trabajadores, mediante cursos de reciclaje, seminarios de capacitación técnica o congresos.

Por último, el perfil periodístico podrá expandirse en la medida en que el propio periodista digital reconozca en el documentalista a un aliado, una parte del equipo con el que debe trabajar coordinadamente. Asimismo, y de igual modo que el periodista adquiere ciertas habilidades documentales, el documentalista deberá reivindicar su espacio para elaborar productos informativos, en los que pueda aparecer en un momento dado su propia firma o la del servicio de documentación, como detalle que contribuya a la visibilidad de las habilidades periodísticas del emisor de la documentación.

\section{Notas}

(1) La contribución que a continuación presentamos constituye uno de los resultados parciales obtenidos por la autora en su tesis doctoral, que con el título La documentación informativa en el periodismo digital: análisis del servicio documental, del documentalista y del mensaje documental y bajo la dirección del profesor Gabriel Galdón, fue defendida en la Universidad Pontificia de Salamanca el 20 de enero de 2004.

(2) Para llevar a cabo este análisis se ha optado por emplear la metodología cualitativa de las entrevistas en profundidad, hechas a documentalistas del servicio de documentación de un medio digital. Estas entrevistas, mantenidas en mayo de 2002, sirven de complemento a la observación directa no participante que también se elaboró en dicho servicio documental, con el objeto de aprovechar el rapport alcanzado con los informantes durante el periodo de observación, garante de la naturalidad y de la confianza necesaria para la conversación. Las entrevistas se han grabado para registrar fielmente las palabras de los documentalistas. Simultáneamente, se ha tomado notas de sus palabras, para anticiparnos ante cualquier fallo técnico de la grabación y recordar los temas relevantes apuntados por los entrevistados. Una vez elaboradas las entrevistas, que se extendieron aproximadamente a una hora de conversación cada una, se han transcrito para facilitar el manejo de los datos. A partir de ahí, el siguiente paso ha sido la lectura detenida de las entrevistas, para extraer de sus comentarios los rasgos que estructuran el perfil del documentalista digital. Finalmente, hemos intercalado en ciertos casos las palabras literales de los documentalistas, cuando estas ayudaban a reforzar la reflexión teórica que tratamos de construir.

Scire. 11 : 2 (sep.-dic. 2005) 17-32. ISSN 1135-3761. 
(3) Este perfil viene a cumplir con una de las recomendaciones propuestas por la SLA a la hora de definir las competencias profesionales del documentalista de la sociedad de la información: "Usa la tecnología de la información apropiada para adquirir, organizar y difundir información”. Y, asimismo, su adopción por parte de los emisores de la documentación digital refleja otra de las cualidades personales sugeridas por la asociación: "Está comprometido con un aprendizaje continuo y con el desarrollo de su carrera profesional” (SLA, 1996).

\section{Referencias}

Galdón López, Gabriel (2002). Funciones informativas de la documentación periodística. // Galdón López, Gabriel (coord.). Teoría y práctica de la documentación informativa. Barcelona: Ariel, 2002. 67-88.

Hernández Pérez, Antonio; Caridad Sebastián, Mercedes (2000). Documentación escrita en los centros de documentación de los medios de comunicación. // Moreiro, José Antonio (coord.). Manual de documentación informativa. Madrid: Cátedra, 2000. 37-82

Paul, Nora (1997a). Information strategy in newsrooms: new emphasis on traditional roles for news librarians. // Poynter Institute. 1997. URL: <http://www.poynter.org/research/nr/nr_strategies.htm>. Consultado: 2002-07-30.

Paul, Nora (1997b). Media libraries and new media. // Poynter Institute. 1997. URL: <http://www.poynter.org/research/nr/nr_libnm.htm>. Consultado: 2002-07-30.

Salvador Oliván, José Antonio; Angós Ullate, José M. ${ }^{\text {a; }}$ Fernández Ruiz, M. ${ }^{a}$ Jesús (1998). El presente y futuro del profesional de la información como intermediario. // El Profesional de la Información. 7:11 (1998) 4-8.

Special Libraries Association (1996). Competencies for Special Librarians of the 21st Century. 1996. URL: <http://www.sla.org/content/SLA/professional/meaning/comp.cfm>. Consultado: 2002-07-30. 